\title{
Appendiceal Neoplasms: Diagnosis, Management and Follow-up
}

\author{
Ana Pereira ${ }^{1 *}$, Joaquim Costa Pereira ${ }^{2}$, Sandra Martins ${ }^{3,4}$ \\ ${ }^{1}$ Surgery Resident, Braga Hospital, 4710-243 Braga, Portugal. \\ ${ }^{2}$ Surgery Department, Coloproctology Unit, Braga Hospital, 4710-243 Braga, Portugal. \\ ${ }^{3}$ Surgery Department, Coloproctology Unit, Braga Hospital, 4710-243 Braga, Portugal; School of Medicine, University of Minho, 4710-070 \\ Braga, Portugal. \\ ${ }^{4}$ Life and Health Sciences Research Institute (ICVS)/3B's - PT Government Associate Laboratory,4710-070 Braga/Guimarães, Portugal.
}

Received 16 February 2021; Revised 06 June 2021; Accepted 22 June 2021; Published 01 September 2021

\begin{abstract}
Appendicectomy is one of the most performed surgical procedures. Neoplasms of the appendix are identified in approximately $1 \%$ of appendiceal specimens, and the incidence seems to be rising. The aim of this review was to summarize the current data on these neoplasms, focusing on the recent classification, clinical manifestations, management, and follow-up of such patients, which remain under debate. A literature search was performed using the database PubMed. The keywords used were related to appendiceal neoplasms, mucocele, pseudomyxoma peritonei and treatment. Records without abstracts, case reports, opinion articles and experimental studies were excluded. The appendix tumors can be classified between epithelial or nonepithelial. The epithelial variant is composed by mucinous neoplasms, nonmucinous adenocarcinoma, and signet ring cell tumors; instead, neuroendocrine tumors, lymphoma, and sarcoma are included in nonepithelial neoplasia. Goblet cell carcinoids share characteristics of both epithelial and nonepithelial tumors. The clinical presentation can be variable and the algorithm for evaluation and treatment is complex. Treatment is based on stage and histology. Our intention is to clarify some questions and help the surgeon in operative decisions, treatment strategies, and patient counseling.
\end{abstract}

Keywords: Appendiceal Neoplasms; Mucocele; Appendiceal Neuroendocrine Tumors; Pseudomyxoma Peritonei.

\section{Introduction}

The appendix was first described in literature in 1522 by Jacopo Berengario da Carpi [1, 2]. However, during the next three centuries, its role in the inflammation of the right lower quadrant was not completely understood [3, 4]. Therefore, it was just in 1735, that Claudius Amyand performed the first appendicectomy that was popularized, as we know it today, by McBurney in 1889 [5]. The number of appendectomies performed increased considerably during the end of the 19th century [2]. It was during that time that the concept of appendiceal neoplasia started to emerge.

Nowadays, over 300,000 appendectomies are performed in the United States per year [6, 7]. With these numbers, it is reasonably that a percentage of these specimens will show unexpected neoplastic pathology. Most of the neoplasms of the appendix are discovered incidentally in the pathological analysis; on the other hand, some can be identified intraoperatively [8].

\footnotetext{
* Corresponding author: anamaria.fppereira@gmail.com

\section{doi http://dx.doi.org/10.28991/SciMedJ-2021-0303-9}

$>$ This is an open access article under the CC-BY license (https://creativecommons.org/licenses/by/4.0/).

(C) Authors retain all copyrights. 
The presence of neoplasia process changes the way we investigate the patient and frequently transform the treatment algorithm. Knowing about the management of appendiceal neoplasms is fundamental to the surgeon. During the time of diagnosis, $74 \%$ of appendiceal cancers have already spread, and more than one-third have regional or distant metastases [9], therefore, decisions about the surgical management can have significant effects on outcome.

The appendix tumors can be classified between epithelial or nonepithelial. The epithelial variant is composed by mucinous neoplasms, nonmucinous adenocarcinoma, and signet ring cell tumors; instead, neuroendocrine tumors, lymphoma, and sarcoma are included in nonepithelial neoplasia. Goblet cell carcinoids share characteristics of both epithelial and nonepithelial tumors.

\section{Epidemiology}

Neoplasms of the appendix are identified in approximately $1 \%$ of appendectomy specimens and its incidence seems to be increasing [9-11]. It has long been stated that neuroendocrine tumors are the most common neoplastic process of the appendix; however, recent statistics have proposed a shift in epidemiology.

The appendix is the third most common location for gastrointestinal neuroendocrine tumors, about $16.7 \%$ of the cases. The most common sites are the small intestine and rectum (44.7 and 19.6\%, respectively) [12]. The appendiceal neuroendocrine tumors (ANETs) are more common in younger patients (32-42 years of age) and are slightly more frequent in females [13]. On the other hand, epithelial tumors of the appendix represent only $0.1 \%$ of epithelial malignancies of the colon and rectum. They are frequently diagnosed in the seventh decade of life and are also more common in females [7-9].

\section{Pathology}

The appendix has a layered wall identical to the rest of the colon. However, the immunological or lymphoid tissue represents a higher percentage of submucosa composition. Furthermore, the goblet cells exist in large numbers and secrete 2 to $3 \mathrm{~mL}$ of mucin every day.

The appendix has only one luminal communication with the colon and that is why an occlusion can end in appendicitis. For this reason, the appendiceal neoplasms usually present clinically as appendicitis, however, less than $50 \%$ of these tumors are recognized during the surgery [8].

\section{Classification}

Appendiceal tumors can be categorized as epithelial or nonepithelial (Figure 1). Epithelial tumors include mucinous neoplasms, nonmucinous adenocarcinoma, and signet ring cell tumors; nonepithelial neoplasms comprise neuroendocrine tumors, lymphoma, and sarcoma. Goblet cell carcinoids share characteristics of both epithelial and nonepithelial tumors.

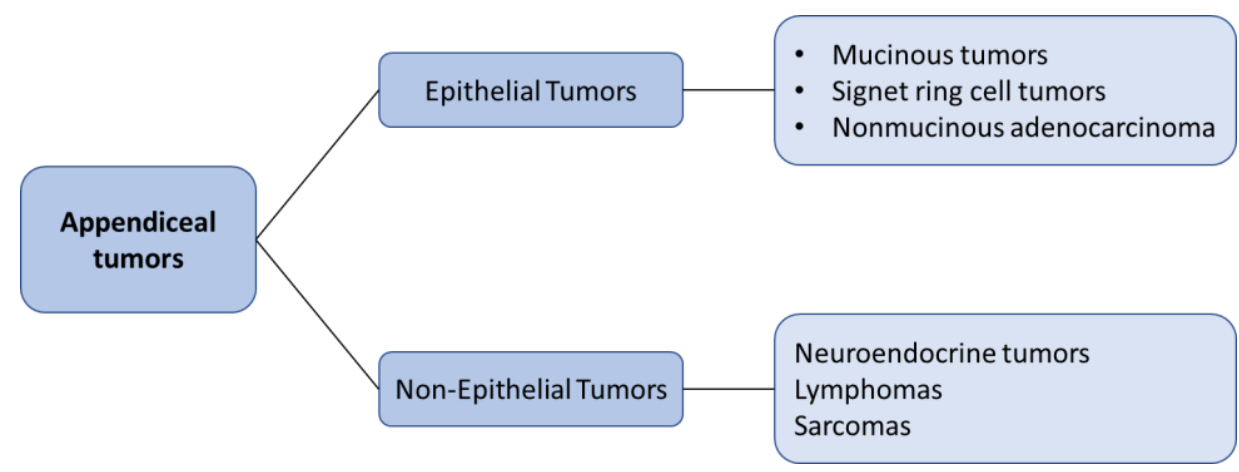

Figure 1. Classification of appendiceal tumors

\subsection{Epithelial Tumors}

The constant shifting of histologic terminology of epithelial neoplasms can create some confusion. However, a recent consensus reported a standardized classification that is presented in Table $1[14,15]$.

\section{Mucinous}

Mucinous tumors can be labeled by grade. Low-grade appendiceal mucinous neoplasms (LAMN) is the generic histologic term for appendiceal adenomas, cystadenomas, borderline tumors, and mucinous tumors of uncertain malignant potential. The critical feature of LAMN is the low-grade cytologic atypia without signs of invasive infiltration in the appendiceal wall [16]. LAMNs are characterized by slow growth, well-differentiated lesions and 
cystic dilation of the appendix associated with fibrosis of the appendiceal wall. Histologically, the normal mucosa is replaced by adenomatous proliferation of villous, papillary, serrated, or flat mucinous lesions. Epithelial cells have basally oriented and small nuclei that are quite uniform, discreet nucleoli and rare mitoses. The cytoplasm frequently comprises abundant mucin resulting in low nuclear to cytoplasmic ratios $[8,16]$.

High-grade appendiceal mucinous neoplasms (HAMN) invade beyond the muscularis mucosa and can be reported as mucinous adenocarcinoma or cystadenocarcinoma. Microscopic inspection shows malignant glandular epithelial cells in strips, clusters, and complex proliferations. High grade dysplasia features cribriform growth, loss of nuclear polarity, enlarged and prominent nucleoli and numerous or atypical mitotic figures [8, 16-17]. It should be mentioned that if HAMN is identified, great care should be taken to exclude the presence of associated invasive adenocarcinoma [18].

The signet ring cell carcinoma is an uncommon variant of mucinous adenocarcinoma. It represents about $4 \%$ of appendiceal tumors and histologically we can notice mucin-laden malignant cells (signet cells) floating in pools of extracellular mucin. These tumors are aggressive and rapidly disseminate. As a result, approximately $60 \%$ have distant metastases at the time of diagnosis $[8,11,19]$.

Regardless the tumor grade, mucocele refers to dilation of the appendix due to the accumulation of mucin. The rupture of the appendix leads to the release of mucin into the peritoneal cavity which can cause mucinosis, pseudomyxoma peritonei (PMP), and peritoneal carcinomatosis [20, 21].

Table 1. Classification of epithelial neoplasms of the appendix and pseudomyxoma peritonei

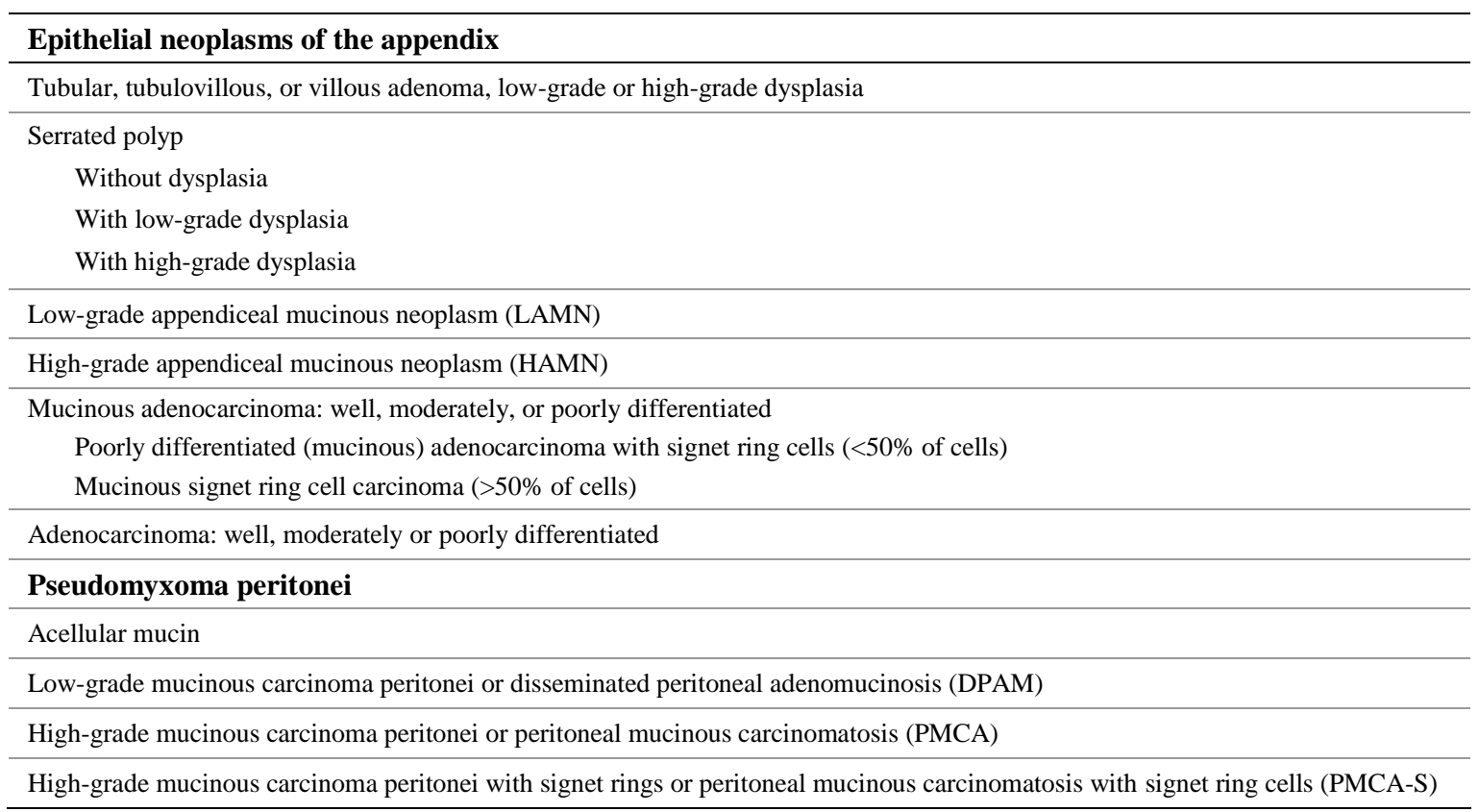

\section{Pseudomyxoma Peritonei}

The rupture of a mucinous appendiceal neoplasm leads to a peritoneal surface disease with secondary production of mucin, which can result in a pseudomyxoma peritonei (PMP). This disease is most caused by an appendiceal primary cancer; however, mucinous tumors of the ovary or pancreas have also been documented.

Solid organ and lymphovascular invasion are rare, nevertheless, the tumors cause fibrosis of tissues and the mucin they produce will fill the abdominal cavity. This will result in death, due to obstruction of abdominal viscera [22]. The prognosis is stated by the malignant potential of the mucinous tumor cells and the extent of peritoneal involvement which is graded by the peritoneal carcinomatosis index (PCI). PCI evaluates the presence and extent of disease in each of nine abdominal areas and 4 small bowel segments [23].

Low-grade mucinous carcinoma peritonei or disseminated peritoneal adenomucinosis (DPAM) usually result from LAMN and most of the times are localized in the right lower abdominal quadrant. These are implants of mucin comprising nonstratified, simple columnar and cuboidal epithelium with uniform nuclei and few mitotic figures [8, 14, 23]. On the other hand, high-grade mucinous carcinoma peritonei or peritoneal mucinous carcinomatosis (PMCA) has a worse response to treatment and prognosis [23]. Microscopic inspection of the implants reveals severe cytologic atypia with enlarged nuclei and prominent nucleoli (like intestinal-type mucinous adenocarcinoma). For this reason, PMCA tend to follow the conduct of peritoneal carcinomatosis from other gastrointestinal cancers, including lymphovascular invasion [24]. 


\section{Nonmucinous}

Nonmucinous appendiceal neoplasms are similar to other primary colorectal adenomatous neoplasia, with comparable natural history and treatment. They progress via direct extension and metastasizes to regional lymph nodes and the liver [25]. Histologically, it can be found glandular formations with relative cellular disorganization and increased stratification [8].

\subsection{Nonepithelial Tumors}

\section{Neuroendocrine}

The appendiceal neuroendocrine tumors (ANETs) were previously called carcinoids. The origin of these tumors are neuroendocrine progenitor cells in the submucosa and most of them $(75 \%)$ are in the appendiceal tip, because of the abundance of subepithelial neuroendocrine cells at the apex of the appendix [26, 27]. In contrast to other gastrointestinal neuroendocrine tumors, ANETs are frequently hormonally active. They can secrete growth hormone (GH), GH-releasing hormone, gastrin, calcitonin, substance $\mathrm{P}$, insulin, neurotensin and serotonin. The clinical manifestations of these hormones are generally blocked by hepatic metabolism; however, liver metastases let enter the systemic circulation and may result in hormonally symptomatic disease. 5- hydroxyindoleacetic acid (5-HIAA) is a product of hepatic metabolism of serotonin which is secreted in the urine and can be useful as tumor marker [8].

Neuroendocrine tumors are well differentiated and relatively indolent [7, 11, 24]. Macroscopically, they appear as yellow-tan, firm nodules, and most of them are less than $1 \mathrm{~cm}$. Microscopic inspection shows conglomerates of cells in the submucosa with an insular pattern and the cytoplasm has a modestly eosinophilic, fine granularity [28].

Chromogranin A is extremely helpful as it may predict early relapse. The Ki-67 protein is also important as a predictor of proliferative capacity and grade of the tumor. A high Ki-67 index is indicative of an aggressive tumor and is accompanied by worse prognosis [28-30].

\section{Lymphoma/Sarcoma}

The appendix has a high percentage of lymphoid tissue, so is not a surprise that lymphoma can occur in this location. However, lymphomas and sarcomas are extremely rare in comparison to other appendiceal neoplasms. Lymphoma of the appendix is a little more prevalent in men $(1.5: 1)$ and the mean age of diagnosis is 18 years old. The main etiology is Burkitt's lymphoma but the elder can develop diffuse, large B cell lymphoma [31].

\subsection{Goblet Cell}

Goblet cell tumors share features of both neuroendocrine and epithelial neoplasms. They are more common in the sixth decade and occur predominantly in females $(4: 1)[32,33]$. They are aggressive tumors with worse prognosis than ANETs and could behave more like appendiceal adenocarcinomas in terms of lymph node involvement, chance of peritoneal spread, and prognosis. Staging, treatment and follow-up of a Goblet cell carcinoma in presence of negative prognostic factors, probably requires a more aggressive approach similar to that of adenocarcinomas [34-36].

\section{Clinical Manifestations}

The clinical presentation of appendiceal neoplasms can be variable. With slowly distention of the appendix, because of the accumulation of mucin, the presenting complaint may be subtle and manifest as a vague lower abdominal pain, due to the stretching of the visceral peritoneum. These situations generally represent mucoceles. Rupture of a mucinous tumor of the appendix can cause PMP and peritoneal carcinomatosis. PMP may become symptomatic just at the end stages of the disease, due to abdominal distention [21]. In the same way, nonmucinous neoplasms can be asymptomatic or associated with ambiguous symptoms, like weight loss or chronic iron deficiency anemia. Finally, even ANETs that are hormonally active can be surreptitious because serotonin syndrome (paradoxical high body temperature, agitation, increased reflexes, tremor, sweating, dilated pupils, or diarrhea) is present in less than $5 \%$, and is generally related to liver metastases and bypass of first-pass metabolism [8].

Apart of the pathologic etiology, an acute appendicitis-like presentation with right lower quadrant pain secondary to distention of the appendix by mucin is the most common clinical presentation in early-stage disease. A neoplastic process in the body or base of the appendix can cause obstruction of the lumen, leading to distention, venous engorgement, and finally infection, with right lower quadrant abdominal pain and appendiceal inflammation on imaging. In these cases, a neoplastic etiology is not usually suspected intraoperatively. However, some factors should increase the surgeon's suspicion for a neoplastic process: age older than 50 years, family history of colon cancer or inflammatory bowel disease, "chronic appendicitis," unexplained anemia, dilated appendix in the absence of appendiceal wall thickening (i.e., $<6 \mathrm{~mm})[8]$. 


\section{Diagnosis and Staging}

Clinical investigation of appendiceal neoplasms depends on where in the algorithm the patient is found. When the symptoms resemble appendicitis, a preoperative imaging is generally done, and then an appendix specimen is taken, permitting a pathologic diagnosis. In other situations, the suspicion can be caused by an incidentally found appendiceal dilation on imaging made for other complaints. Despite all of this, computed tomography (CT) or magnetic resonance imaging (MRI) of the abdomen is essential. Imaging studies is also important to dismiss metastatic disease. In cases of adenocarcinoma, a CT of the chest is crucial to rule out pulmonary metastases.

Mucoceles usually manifest as appendiceal dilation $>15 \mathrm{~mm}$ without adjacent inflammation [37]. Peritoneal involvement can also be diagnosed based on physical examination and cross-sectional imaging. The abdomen is usually distended, dull to percussion, and nontender. Imaging can show hypoattenuating ascites with secondary signs of extrinsic compression, like scalloping of the liver, peritoneal reflection, or pouch of Douglas nevertheless, these are normally late findings [8].

Regarding tumor markers, baseline carcinoembryonic antigen (CEA) may be measured for adenocarcinoma, while chromogranin A and Urine 5-HIAA must be obtained for neuroendocrine tumors. A complete colonoscopy should also be performed since synchronous colon lesions have a 10 to $20 \%$ incidence in both epithelial and neuroendocrine tumors [38]. Positron emission tomography (PET) may be a practical complement in cases with suspicious lesions on more conventional imaging [8].

\section{Surgical Management}

Surgery is the main treatment for almost all appendiceal neoplasms, with exception of lymphoma, advanced PMP (Peritoneal Carcinomatosis Index [PCI] > 22), neuroendocrine tumors with systemic metastases, or adenocarcinoma with both peritoneal disease and either supradiaphragmatic or multiple solid organ metastases.

A great number of appendiceal neoplasms are identified just when the pathologic analysis is completed. When appendectomy alone is not adequate, additional actions may be necessary to accomplish a complete staging and treatment. During the appendectomy, if the surgeon has a high suspicion but the base of the appendix is free of tumor, it is not incorrect to do the appendectomy as a diagnostic excisional biopsy and make additional plans according to the definitive diagnosis. On the other hand, if the base of the appendix/ cecum is involved, it should be done an oncologic colectomy.

\subsection{Appendectomy}

The appendectomy alone is the appropriate treatment in some situations such as adenomatous polyps, adenocarcinoma in situ, or nonperforated low-grade mucinous tumors with R0 margins. Concerning neuroendocrine tumors, subcentimeter, isolated tumors with an R0 margin are also completely treated by appendectomy. Even neuroendocrine tumors with $1-2 \mathrm{~cm}$ may be treated with an appendectomy alone, if the margin is clear, there is less than $3 \mathrm{~mm}$ of mesoappendiceal invasion, multifocality is not present, and there is no involvement of regional nodal or isolated liver [29, 39]. In all these situations the surveillance colonoscopy should be performed in the case of pre or early malignancy.

\subsection{Right Hemicolectomy}

Right colectomy with lymphadenectomy should be performed when there is involvement of the cecum; in situations such as high-grade neoplasm or adenocarcinoma; tumor size greater than $2 \mathrm{~cm}$; goblet cell tumor; or neuroendocrine tumor $>1-2 \mathrm{~cm}$, invading mesoappendix or regional nodal [40, 41].

\subsection{Cytoreductive Surgery and Hyperthermic Intraperitoneal Chemotherapy}

Appendiceal mucinous neoplasm should have a special attention, as PMP is frequent after resection, particularly if there is perforation, and can occur 10 years after the appendectomy. When extra-appendiceal spread of mucin is present during the appendectomy, the best decision is to stop, biopsy the implants and mucin, register in photos, and refer to a center specializing in management of these tumors [40]. Patients with evidence of accumulation of peritoneal mucin necessitates consultation to a hyperthermic intraperitoneal chemotherapy (HIPEC) and a precise diagnosis is associated with the number of biopsies [23]. Since the 1980's, the introduction and widespread use of cytoreductive surgery and perioperative HIPEC has provided a treatment option improving prognosis, outcome, and quality of life with potential for cure for patients with PMP [42, 43]. If there is perforation, an appendectomy should be done to control the contamination, but it is imprudent to manipulate the abdominal viscera, because it can compromise the following cytoreductive surgery. 
Patients with high-grade appendiceal neoplasms and peritoneal dissemination have some treatment options that comprise chemotherapy, cytoreductive surgery or HIPEC. After staging and colonoscopy, the target is to remove the visible tumor, leaving HIPEC for the remaining microscopic disease. The aim of HIPEC is to deliver a regionally high intraperitoneal dose of heated chemotherapy with minimal systemic effects. A high dose of heated chemotherapeutic agents is perfused intraoperatively throughout the abdomen to eradicate any residual microscopic cancer cells. Therefore, when a complete or near complete cytoreduction is obtained (when all tumour nodules bigger than $0.25 \mathrm{~cm}$ have been removed), HIPEC is added to treat microscopic residual tumour cells [44].

The HIPEC surgeon calculate the PCI during the laparotomy. PCI is a prognostic tool that is a sum of scores in thirteen abdominal regions. Each receives a score of 0-3 based on the largest tumor size in each region. Scores range from 0 to 39. Cytoreductive surgery and HIPEC is most effective when the PCI is low, tumors are low grade, and complete or near complete cytoreduction is accomplished [41, 45]. Various studies have shown that survival is improved with low-grade PMP compared to highgrade PMP [46, 47]. Unresectable cases include PMP from adenocarcinoma with a PCI > 18 and PMP with simultaneous systemic metastases [8]. The radiologist must also recognize what constitutes a resectable or unresectable disease at cytoreduction. In the end, the pre-operative assessment of patients with peritoneal carcinomatosis involves a multi-specialty team approach [48].

\subsection{Preoperative Systemic Chemotherapy}

Optimal cytoreductive surgery and HIPEC treatment without preoperative systemic chemotherapy remains the standard of care in the management of peritoneal metastasis of LAMN. High-grade appendiceal adenocarcinomas should be considered for systemic preoperative chemotherapy and if there is response, surgical resection and HIPEC treatment should be considered [41,49].

\section{Follow-up}

The surveillance of mucinous or nonmucinous adenocarcinoma of appendix is supposed to follow colorectal cancer protocols. This should consist of CEA levels every 3 months for 2 years and every 6 months for the following 3 years; $\mathrm{CT}$ of the chest, abdomen, and pelvis should be performed biannually for 5 years and colonoscopy should be done at years 1 and 3, and then every 5 years, depending on findings [50].

Concerning to nonperforated LAMN, the surveillance after resection is controversial, but generally it can be followed with a colonoscopy one year after resection and no further imaging. If LAMN is perforated or has extracellular mucin, a CT or MRI should be performed 6-12 months after the appendectomy. If these exams show no mucin, additional follow up may not be needed, although some centers continue to follow these patients for 5 years or longer [51]. Regarding neuroendocrine tumors, if these are less than $2 \mathrm{~cm}$ without high-risk features, a R0 resection is curative and the patients do not need specific follow-up. Tumors more than $2 \mathrm{~cm}$ or with high-risk features require CT abdomen and pelvis, and tumor marker levels every 6 to 12 months for at least 7 years after surgery [52].

\section{Prognosis}

The outcomes of appendiceal neoplasia are variable and depends on the etiology and stage of tumor. Depending on the histological type, the 5-year disease survival goes from 27 to $93 \%$. The signet ring cell tumors have the worst prognosis and the neuroendocrine have the most positive outcomes [53]. The mucinous carcinomatosis have an unkind prognosis with less than $10 \%$ of 5-year survival rates [22]. The combination of cytoreductive surgery and HIPEC, in selected patients with PMP, is believed to improve 5- and 10-year survival rates to $72 \%$ and 54\%, respectively [54].

The size of neuroendocrine tumors is an important prognosis indicator in what concerns to regional and distant spread. Tumors $<1 \mathrm{~cm}$ have rarely spread at the time of diagnosis and have 5-year survival rates close to $100 \%$. On the other and, tumors $>2 \mathrm{~cm}$ have a 20 to $30 \%$ rate of nodal or distant spread, and a 5-year survival rate around $31 \%$ [39, 55]. Concerning goblet cell carcinoids, at least $10 \%$ tumors have widespread metastases at the time of diagnosis. The 5-year survival rates are 100, 76, 22, and 14\% for stages I, II, III, and IV disease, respectively [32].

\section{Conclusions}

Neoplasms of the appendix are identified in approximately $1 \%$ of appendectomy specimens and its incidence seems to be increasing. They can be classified between epithelial or nonepithelial. The clinical presentation is variable but, apart of the pathologic etiology, appendicitis is the most acute manifestation of appendiceal neoplasia. The algorithm for evaluation and treatment is complex and relies strongly on often subtle histopathologic difference.

Mucinous tumors of the appendix and PMP have created a lot of curiosity in recent years due to the application of radical surgical procedures which result in a prolonged survival. Tumors restricted to the appendiceal mucosa are treated by simple excision of the appendix while those beyond the mucosa with epithelial proliferation are associated with dissemination of the disease. Histological grade is essential as tumors with a low grade are managed with cytoreductive surgery and HIPEC alone while those with high grade disease might also require preoperative systemic 
chemotherapy. When extra-appendiceal spread of mucin is present during the appendectomy, the best decision is to stop, biopsy the implants and mucin, register in photos, and refer to a specialized center. It is important to decide and discuss the proper course of action with the patient and provide an accurate estimate of the risks and benefits of the treatment or surveillance. Multidisciplinary teamwork is required to provide each patient the most appropriate, personalized treatment plan. The surgeon must be comfortable with the treatment algorithm for this spectrum of disease. This can make a significant difference in the management and the prognosis of these patients.

\section{Declarations}

\subsection{Author Contributions}

Conceptualization, A.P., J.C.P. and S.M.; methodology, A.P., J.C.P. and S.M.; validation, A.P. and S.F.M.; formal analysis, A.P.; investigation, A.P.; writing — original-draft preparation, A.P.; writing - review and editing, A.P., J.C.P. and S.F.M.; supervision, J.C.P. and S.F.M.; project administration, S.F.M. All authors have read and agreed to the published version of the manuscript.

\subsection{Funding}

The authors received no financial support for the research, authorship, and/or publication of this article.

\subsection{Ethical Approval}

This review contains no experiments on humans and animals, so ethical approval not required.

\subsection{Data Availability Statement}

Data sharing is not applicable to this article.

\subsection{Conflict of Interest}

The authors declare that they have no known competing financial interests or personal relationships that could have appeared to influence the work reported in this paper.

\section{References}

[1] Deaver, J. B. (1905). Appendicitis. Philadelphia: P Blakiston's Son \& Co.

[2] Williams, G. R. (1983). Presidential Address: A History of Appendicitis. Annals of Surgery, 197(5), 495-506. doi:10.1097/00000658-198305000-00001.

[3] Cope, Z. (1965). A history of the acute abdomen. Oxford University Press, USA.

[4] Major, R. H. (1945). Classic descriptions of disease: with biographical sketches of the authors. Charles C Thomas Pub Limited.

[5] McBurney, C. M. (1889). Experience with early operative interference in cases of disease of the vermiform appendix. New York State Journal of Medicine, 50, 676-684.

[6] Barrett, M. L., Hines, A. L., \& Andrews, R. M. (2013). Trends in rates of perforated appendix, 2001-2010: statistical brief\# 159. Available online: https://www.hcup-us.ahrq.gov/reports/statbriefs/sb159.pdf (accessed on May 2021).

[7] McCusker, M. E., Coté, T. R., Clegg, L. X., \& Sobin, L. H. (2002). Primary malignant neoplasms of the appendix. Cancer, 94(12), 3307-3312. doi:10.1002/cncr.10589.

[8] Spanos, C. P., \& Kaiser, A. M. (2016). Appendiceal Neoplasms. The ASCRS Textbook of Colon and Rectal Surgery, 617-629. doi:10.1007/978-3-319-25970-3_37.

[9] Marmor, S., Portschy, P. R., Tuttle, T. M., \& Virnig, B. A. (2015). The Rise in Appendiceal Cancer Incidence: $2000-2009$. Journal of Gastrointestinal Surgery, 19(4), 743-750. doi:10.1007/s11605-014-2726-7.

[10] Whitfield, C. G., Amin, S. N., \& Garner, J. P. (2012). Surgical management of primary appendiceal malignancy. Colorectal Disease, 14(12), 1507-1511. doi:10.1111/j.1463-1318.2012.03052.x.

[11] McGory, M. L., Maggard, M. A., Kang, H., O’Connell, J. B., \& Ko, C. Y. (2005). Malignancies of the Appendix: Beyond Case Series Reports. Diseases of the Colon \& Rectum, 48(12), 2264-2271. doi:10.1007/s10350-005-0196-4.

[12] Maggard, M. A., O??Connell, J. B., \& Ko, C. Y. (2004). Updated Population-Based Review of Carcinoid Tumors. Annals of Surgery, 240(1), 117-122. doi:10.1097/01.sla.0000129342.67174.67.

[13] Alexandraki, K. I., Kaltsas, G. A., Grozinsky-Glasberg, S., Chatzellis, E., \& Grossman, A. B. (2015). Appendiceal neuroendocrine neoplasms: diagnosis and management. Endocrine-Related Cancer, 23(1), R27-R41. doi:10.1530/erc-15-0310.

[14] Carr, N. J., Cecil, T. D., Mohamed, F., Sobin, L. H., Sugarbaker, P. H., González-Moreno, S., ... Moran, B. J. (2016). A Consensus for Classification and Pathologic Reporting of Pseudomyxoma Peritonei and Associated Appendiceal Neoplasia. American Journal of Surgical Pathology, 40(1), 14-26. doi:10.1097/pas.0000000000000535. 
[15] Carr, N. J., Bibeau, F., Bradley, R. F., Dartigues, P., Feakins, R. M., Geisinger, K. R., ... Yantiss, R. K. (2017). The histopathological classification, diagnosis and differential diagnosis of mucinous appendiceal neoplasms, appendiceal adenocarcinomas and pseudomyxoma peritonei. Histopathology, 71(6), 847-858. doi:10.1111/his.13324.

[16] Legué, L. M., Creemers, G.-J., de Hingh, I. H. J. T., Lemmens, V. E. P. P., \& Huysentruyt, C. J. (2019). Review: Pathology and Its Clinical Relevance of Mucinous Appendiceal Neoplasms and Pseudomyxoma Peritonei. Clinical Colorectal Cancer, 18(1), 1-7. doi:10.1016/j.clcc.2018.11.007.

[17] Pai, R. K., Beck, A. H., Norton, J. A., \& Longacre, T. A. (2009). Appendiceal Mucinous Neoplasms. American Journal of Surgical Pathology, 33(10), 1425-1439. doi:10.1097/pas.0b013e3181af6067.

[18] Valasek, M. A., \& Pai, R. K. (2018). An Update on the Diagnosis, Grading, and Staging of Appendiceal Mucinous Neoplasms. Advances in Anatomic Pathology, 25(1), 38-60. doi:10.1097/pap.0000000000000178.

[19] Ruoff, C., Hanna, L., Zhi, W., Shahzad, G., Gotlieb, V., \& Saif, M. W. (2011). Cancers of the Appendix: Review of the Literatures. ISRN Oncology, 2011, 1-6. doi:10.5402/2011/728579.

[20] Sugarbaker, P. H. (2006). The natural history, gross pathology, and histopathology of appendiceal epithelial neoplasms. European Journal of Surgical Oncology (EJSO), 32(6), 644-647. doi:10.1016/j.ejso.2006.03.016.

[21] Sugarbaker, P. H. (2009). Epithelial Appendiceal Neoplasms. The Cancer Journal, 15(3), $225-235$. doi:10.1097/ppo.0b013e3181a9c781.

[22] Compton, C., Fenoglio - Preiser, C. M., Pettigrew, N., \& Fielding, L. P. (2000). American joint committee on cancer prognostic factors consensus conference: colorectal working group. cancer, 88(7), 1739-1757.

[23] Ronnett, B. M., Zahn, C. M., Kurman, R. J., Kass, M. E., Sugarbaker, P. H., \& Shmookler, B. M. (1995). A Clinicopathologic Analysis of 109 Cases with Emphasis on Distinguishing Pathologic Features, Site of Origin, Prognosis, and Relationship to "Pseudomyxoma Peritonei." The American Journal of Surgical Pathology, 19(12), 1390-1408. doi:10.1097/00000478199512000-00006

[24] Pai, R. K., \& Longacre, T. A. (2005). Appendiceal mucinous tumors and pseudomyxoma peritonei: histologic features, diagnostic problems, and proposed classification. Advances in anatomic pathology, 12(6), 291-311. doi:10.1097/01.pap.0000194625.05137.51.

[25] Misdraji, J. (2013). Tumors of the appendix. Morson's and Dawson's Gastrointestinal Pathology. London: Wiley; $490-498$.

[26] Lundqvist, M., \& Wilander, E. (1986). Subepithelial neuroendocrine cells and carcinoid tumours of the human small intestine and appendix. A comparative immunohistochemical study with regard to serotonin, neuron-specific enolase and S-100 protein reactivity. The Journal of Pathology, 148(2), 141-147. doi:10.1002/path.1711480204.

[27] Shaw, P. A. V. (1991). The topographical and age distributions of neuroendocrine cells in the normal human appendix. The Journal of Pathology, 164(3), 235-239. doi:10.1002/path.1711640308.

[28] Komminoth, P., Arnold, R., Capella, C., Klimstra, D. S., Klöppel, G., Solcia, E., \& Rindi, G. (2010). Neuroendocrine neoplasms of the appendix. WHO classification of tumours of the digestive system, 126-128.

[29] Pape, U.-F., Perren, A., Niederle, B., Gross, D., Gress, T., Costa, F., .. Grossman, A. (2012). ENETS Consensus Guidelines for the Management of Patients with Neuroendocrine Neoplasms from the Jejuno-Ileum and the Appendix Including Goblet Cell Carcinomas. Neuroendocrinology, 95(2), 135-156. doi:10.1159/000335629.

[30] Moris, D., Tsilimigras, D. I., Vagios, S., Ntanasis-Stathopoulos, I., Karachaliou, G. S., Papalampros, A., Alexandrou, A., Blazer, D. G., Felekouras, E. (2018). Neuroendocrine Neoplasms of the Appendix: A Review of the Literature. Anticancer Res., 38(2):601-611. doi: 10.21873/anticanres.12264.

[31] Stewart, R. J., \& Mirakhur, M. (1986). Primary malignant lymphoma of the appendix. The Ulster medical journal, 55(2), 187189.

[32] Pham, T. H., Wolff, B., Abraham, S. C., \& Drelichman, E. (2006). Surgical and Chemotherapy Treatment Outcomes of Goblet Cell Carcinoid: A Tertiary Cancer Center Experience. Annals of Surgical Oncology, 13(3), 370-376. doi:10.1245/aso.2006.02.016.

[33] Tang, L. H., Shia, J., Soslow, R. A., Dhall, D., Wong, W. D., O’Reilly, E., ... Klimstra, D. S. (2008). Pathologic Classification and Clinical Behavior of the Spectrum of Goblet Cell Carcinoid Tumors of the Appendix. American Journal of Surgical Pathology, 32(10), 1429-1443. doi:10.1097/pas.0b013e31817f1816.

[34] Yozu, M., Johncilla, M. E., Srivastava, A., Ryan, D. P., Cusack, J. C., Doyle, L., ... Misdraji, J. (2018). Histologic and Outcome Study Supports Reclassifying Appendiceal Goblet Cell Carcinoids as Goblet Cell Adenocarcinomas, and Grading and Staging Similarly to Colonic Adenocarcinomas. American Journal of Surgical Pathology, 42(7), 898-910. doi:10.1097/pas.0000000000001056.

[35] Zhang, K., Meyerson, C., Kassardjian, A., Westbrook, L. M., Zheng, W., \& Wang, H. L. (2019). Goblet Cell Carcinoid/Carcinoma: An Update. Advances in Anatomic Pathology, 26(2), 75-83. doi:10.1097/pap.0000000000000222. 
[36] Nonaka, D., Papaxoinis, G., Lamarca, A., Fulford, P., Valle, J., \& Chakrabarty, B. (2018). A study of appendiceal crypt cell adenocarcinoma (so-called goblet cell carcinoid and its related adenocarcinoma). Human Pathology, 72, 18-27. doi:10.1016/j.humpath.2017.08.005.

[37] Madwed, D., Mindelzun, R., \& Jeffrey, R. B. (1992). Mucocele of the appendix: imaging findings. American Journal of Roentgenology, 159(1), 69-72. doi:10.2214/ajr.159.1.1609724.

[38] Connor, S. J., Hanna, G. B., \& Frizelle, F. A. (1998). Appendiceal tumors. Diseases of the Colon \& Rectum, 41(1), 75-80. doi:10.1007/bf02236899.

[39] Mullen, J. T., \& Savarese, D. M. F. (2011). Carcinoid tumors of the appendix: A population-based study. Journal of Surgical Oncology, 104(1), 41-44. doi:10.1002/jso.21888.

[40] Kusamura, S., Moran, B. J., Sugarbaker, P. H., Levine, E. A., Elias, D., Baratti, D., ... Liauw, W. (2014). Multicentre study of the learning curve and surgical performance of cytoreductive surgery with intraperitoneal chemotherapy for pseudomyxoma peritonei. British Journal of Surgery, 101(13), 1758-1765. doi:10.1002/bjs.9674.

[41] Esquivel, J., Chua, T. C., Stojadinovic, A., Melero, J. T., Levine, E. A., Gutman, M., ... Sticca, R. P. (2010). Accuracy and clinical relevance of computed tomography scan interpretation of peritoneal cancer index in colorectal cancer peritoneal carcinomatosis: A multi-institutional study. Journal of Surgical Oncology, 102(6), 565-570. doi:10.1002/jso.21601.

[42] Stearns, A. T., Malcomson, L., Punnett, G., Abudeeb, H., Aziz, O., Selvasekar, C. R., ... O’Dwyer, S. T. (2018). Long-term Quality of Life After Cytoreductive Surgery and Heated Intraperitoneal Chemotherapy for Pseudomyxoma Peritonei: A Prospective Longitudinal Study. Annals of Surgical Oncology, 25(4), 965-973. doi:10.1245/s10434-017-6326-0.

[43] Lurvink, R. J., Villeneuve, L., Govaerts, K., de Hingh, I. H. J. T., Moran, B. J., Deraco, M., ... Kusamura, S. (2021). The Delphi and GRADE methodology used in the PSOGI 2018 consensus statement on Pseudomyxoma Peritonei and Peritoneal Mesothelioma. European Journal of Surgical Oncology, 47(1), 4-10. doi:10.1016/j.ejso.2019.03.012.

[44] Soucisse, M. L., Lansom, J., Alshahrani, M. S., \& Morris, D. L. (2020). Mucinous appendiceal neoplasms with or without pseudomyxoma peritonei: a review. ANZ Journal of Surgery, 90(10), 1888-1894. doi:10.1111/ans.16185.

[45] Grotz, T. E., Overman, M. J., Eng, C., Raghav, K. P., Royal, R. E., Mansfield, P. F., .. Fournier, K. F. (2017). Cytoreductive Surgery and Hyperthermic Intraperitoneal Chemotherapy for Moderately and Poorly Differentiated Appendiceal Adenocarcinoma: Survival Outcomes and Patient Selection. Annals of Surgical Oncology, 24(9), $2646-2654$. doi:10.1245/s10434-017-5938-8.

[46] Huang, Y., Alzahrani, N. A., Chua, T. C., \& Morris, D. L. (2017). Histological Subtype Remains a Significant Prognostic Factor for Survival Outcomes in Patients With Appendiceal Mucinous Neoplasm With Peritoneal Dissemination. Diseases of the Colon \& Rectum, 60(4), 360-367. doi:10.1097/dcr.0000000000000719.

[47] Gupta, N., Asif, S., Gandhi, J., Rajpurohit, S., \& Singh, S. (2017). Role of CRS and HIPEC in appendiceal and colorectal malignancies: Indian experience. Indian Journal of Gastroenterology, 36(2), 126-130. doi:10.1007/s12664-017-0747-3.

[48] Sugarbaker, P. H., Sardi, A., Brown, G., Dromain, C., Rousset, P., \& Jelinek, J. S. (2017). Concerning CT features used to select patients for treatment of peritoneal metastases, a pictoral essay. International Journal of Hyperthermia, 33(5), $497-504$. doi:10.1080/02656736.2017.1317368.

[49] Sugarbaker, P. H., Bijelic, L., Chang, D., \& Yoo, D. (2010). Neoadjuvant FOLFOX chemotherapy in 34 consecutive patients with mucinous peritoneal carcinomatosis of appendiceal origin. Journal of Surgical Oncology, 102(6), 576-581. doi:10.1002/jso.21679.

[50] Anthony, T., Simmang, C., Hyman, N., Buie, D., Kim, D., Cataldo, P., ... Whiteford, M. (2004). Practice Parameters for the Surveillance and Follow-Up of Patients With Colon and Rectal Cancer. Diseases of the Colon \& Rectum, 47(6), 807-817. doi:10.1007/s10350-004-0519-x.

[51] Guaglio, M., Sinukumar, S., Kusamura, S., Milione, M., Pietrantonio, F., Battaglia, L., ... Deraco, M. (2018). Correction to: Clinical Surveillance After Macroscopically Complete Surgery for Low-Grade Appendiceal Mucinous Neoplasms (LAMN) with or Without Limited Peritoneal Spread: Long-Term Results in a Prospective Series. Annals of Surgical Oncology, 25(S3), 987-987. doi:10.1245/s10434-018-6341-9.

[52] Boudreaux, J. P., Klimstra, D. S., Hassan, M. M., Woltering, E. A., Jensen, R. T., Goldsmith, S. J., ... Yao, J. C. (2010). The NANETS Consensus Guideline for the Diagnosis and Management of Neuroendocrine Tumors. Pancreas, 39(6), $753-766$. doi:10.1097/mpa.0b013e3181ebb2a5.

[53] Turaga, K. K., Pappas, S. G., \& Gamblin, T. C. (2012). Importance of Histologic Subtype in the Staging of Appendiceal Tumors. Annals of Surgical Oncology, 19(5), 1379-1385. doi:10.1245/s10434-012-2238-1.

[54] González-Moreno, S., \& Sugarbaker, P. H. (2004). Right hemicolectomy does not confer a survival advantage in patients with mucinous carcinoma of the appendix and peritoneal seeding. British Journal of Surgery, 91(3), 304-311. doi:10.1002/bjs.4393.

[55] Stinner, B., Kisker, O., Zielke, A., \& Rothmund, M. (1996). Surgical Management for Carcinoid Tumors of Small Bowel, Appendix, Colon, and Rectum. World Journal of Surgery, 20(2), 183-188. doi:10.1007/s002689900028. 